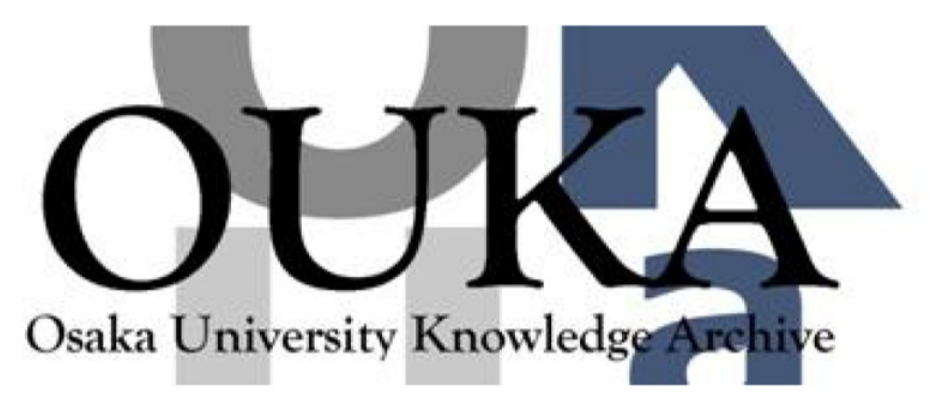

\begin{tabular}{|c|l|}
\hline Title & $\begin{array}{l}\text { Measurement of elastic constant and refraction } \\
\text { index of thin films at low temperatures using } \\
\text { picosecond ultrasound }\end{array}$ \\
\hline Author(s) & $\begin{array}{l}\text { Tanigaki, Kenichi; Kusumoto, Tatsuya; Ogi, } \\
\text { Hirotsugu et al. }\end{array}$ \\
\hline Citation & $\begin{array}{l}\text { Japanese Journal of Applied Physics. 49 } \\
\text { p. } 07 \text { HB01 }\end{array}$ \\
\hline Issue Date & $2010-07-20$ \\
\hline oaire:version & AM \\
\hline URL & https://hdl. handle. net/11094/84164 \\
\hline rights & \\
\hline Note & \\
\hline
\end{tabular}

Osaka University Knowledge Archive : OUKA

https://ir. Library. osaka-u. ac. jp/

Osaka University 


\section{Measurement of Elastic Constant and Refraction Index of Thin Films at Low Temperatures Using Picosecond Ultrasound}

Kenichi TANIGAKI, Tatsuya KUSUMOTO, Hirotsugu OGI*, Nobutomo NAKAMURA, and Masahiko HIRAO

Graduate School of Engineering Science, Osaka University, 1-3 Machikaneyama, Toyonaka, Osaka

560-8531, Japan

In this paper, a picosecond ultrasound measurement is conducted to evaluate low-temperature elasticity of thin films and semiconductors. Specimens are cooled with liquid He through a heat exchanger in a cryostat, and an ultrahigh-frequency acoustic pulse is generated using a femtosecond light pulse, which propagates in the film-thickness direction. One of three phenomena, pulse echoes, acoustic phonon resonances, and Brillouin oscillations, is observed by the reflectivity changes of the time-delayed probe light, depending on the material. They give the longitudinal-wave out-of-plane elastic constant. When the stiffness at low temperature is known, the last phenomenon provides the refractive index. We determined the stiffness of $\mathrm{Pt}$ thin film and refractive index of $\mathrm{Si}$ at $5 \mathrm{~K}$. The methodology developed in this paper is effective to study elastic and optic properties of metallic thin films and transparent materials at low temperatures.

${ }^{*}$ E-mail address: ogi@me.es.osaka-u.ac.jp 


\section{Introduction}

The elastic constants of solids remain the central issue in condensed matter physics, because they directly reflect the interatomic potential. Especially, the elastic property of thin films deserves intensive study because of their unusual behaviors. ${ }^{1,2)}$ Thomsen et. al. first detected ultrahigh frequency acoustic phonons using ultrafast pump-probe light pulses. ${ }^{3,4)}$ Following their work, the picosecond laser ultrasound (PSLU) method was developed for the study of ultrahigh frequency acoustic properties in solids. ${ }^{5-7)}$ In the PSLU method, ultrasound pulses with gigahertz frequency are generated and detected on the picosecond time scale. Therefore, with accurate measurement of film thickness using the x-ray total reflectivity method, ${ }^{8)}$ the PSLU method yields the elasticity of thin films. Using these techniques, we studied elasticity of $\mathrm{Pt},{ }^{1,2)} \mathrm{Fe},{ }^{2)} \mathrm{Cu},{ }^{9)}$ and oxide ${ }^{10,11)}$ thin films. In addition, theoretical analysis using ab-initio calculation is matched up with the measurements of metallic superlattice ${ }^{12)}$ and nanocrystalline diamond films. ${ }^{13,14)}$

These previous studies reveal that some metallic thin films show unusual elastic property. For example, ultrathin Pt thin films show significantly larger stiffness than the bulk material, and its physical reason remains unclear. ${ }^{1)}$ The PSUL method at liquid helium temperature is expected to solve their mechanisms, because the essential physical properties appear at cryogenic temperatures, being free from the thermal disturbance. Therefore, it allows us to compare measurements with theoretical calculations based on ab-initio calculations.

Optical properties of semiconductors are also unknown at cryogenic temperatures. They are needed in the aero-space telescope technology. ${ }^{15)}$ However, the PSLU method can detect the Brillouin oscillation for a semiconductor, whose vibrational frequency depends on the refractive index and the sound velocity of the material. Therefore, we can determine the refractive index when we know the sound velocity at low temperatures. Thus, the PSLU method at low temperatures is very important in advances of nanomechanics and optic technologies. However, it remains at $25 \mathrm{~K}$ conducted by Hao and Maris. ${ }^{16)}$ In this study, we develop the PSLU method for the study of elasticity of thin films at low temperatures down to $5 \mathrm{~K}$.

\section{Experiment}

The PSLU measurement is classified into three methods. First is the pulse-echo method. ${ }^{4-6,12)}$ The acoustic pulse generated by the pump light repeats reflections between the film surface and the film-substrate interface until the energy dissipates. The round-trip time and echo amplitudes were measured by the probe light to determine the sound velocity and attenuation, respectively. The acoustic-phonon resonance spectroscopy ${ }^{1,2,9)}$ is applied to evaluate the elasticity of ultrathin films $(\sim<30 \mathrm{~nm})$, where the standing wave is measured which resonance 
frequency $f$ is associated with the sound velocity $v$;

$$
f= \begin{cases}\frac{m v}{2 d} & \left(Z_{f}>Z_{s}\right) \\ \frac{(2 m-1) v}{4 d} & \left(Z_{s}>Z_{f}\right)\end{cases}
$$

Here, $Z_{f}$ and $Z_{s}$ are acoustic impedance of film and substrate, and $m$ denotes order of resonance mode, respectively. In the case of the transparent or translucent material, the Brillouin oscillation $^{4,7,10,11,13)}$ is observed. It arises from interference between the reflected light from the surface of the specimen and the diffracted light from the acoustic wave. In the first-order approximation for strain, the oscillation frequency $f$ relates with the sound velocity $v$ via Bragg' s condition when the probe light enters the film perpendicularly;

$$
f=\frac{2 n v}{\lambda} \text {. }
$$

Here, $n$ and $\lambda$ denote the refractive index of the examined material and the wavelength of the probe light, respectively. This equation is applicable when the film thickness is larger than three times of the wavelength of the probe light. ${ }^{11}$ ) Thus, the sound velocity is obtained by measuring the Brillouin oscillation frequency when the refractive index is known, and the refractive index is obtained vice versa. The pump-probe method determines the out-of-plane elastic constant from the velocity of the longitudinal wave propagating along the thickness direction.

In this study, we develop an optics, which is equipped with a cryostat, as shown in Fig. 1. We irradiate a second-harmonic-generator (SHG) crystal with a titanium-sapphire pulse laser at $800 \mathrm{~nm}$ wavelength and $140 \mathrm{fs}$ pulse width. The SHG generates the frequency-doubled pulse, which are separated into the pump light $(\lambda=800 \mathrm{~nm})$ and the probe light $(\lambda=400$ $\mathrm{nm})$ by a dichroic mirror. The former is modulated by an acousto-optic modulator $(\mathrm{A} / \mathrm{O})$ with the modulation frequency of $100 \mathrm{kHz}$, and its first order diffraction light is focused on the specimen to generate the acoustic pulse through thermal expansion. The latter is delayed by moving of a corner reflector and perpendicularly focused on the same spot on the specimen as the probe light for detection. The reflectivity change of the probe light is then detected by a balanced detector, and the $100 \mathrm{kHz}$ component of the reflectivity change is extracted using a lock-in amplifier. The specimen is held in the cryostat and cooled by liquid helium through a $\mathrm{Cu}$ heat exchanger down to liquid helium temperature. The pressure inside the head of the cryostat is maintained at $10^{-3} \mathrm{~Pa}$ by the diffusion pump, achieving the effective thermal insulation. The temperature, stage position, data acquisition, and the flow rate of the liquid helium are controlled by a personal computer. 


\section{Result and Discussion}

\subsection{Pt thin film on Si substrate}

We deposited Pt thin film by RF magnetron sputtering on (001) face of a monocrystal Si substrate. Before the deposition, the substrate surface was cleaned by a piranha solution $\left(98 \% \mathrm{H}_{2} \mathrm{SO}_{4}: 33 \% \mathrm{H}_{2} \mathrm{O}_{2}=7: 3\right)$, and rinsed by ultrapure water several times. The film thickness is $65.1 \mathrm{~nm}$, which is determined by the x-ray total reflectivity measurement(Fig. 2). The highangle x-ray diffraction (XRD) measurement using $\mathrm{CoK} \alpha$ radiation was performed to evaluate the crystallographic orientation of the film. The XRD spectra indicate strong (111) texture for Pt; diffraction peaks from other planes were negligible, as shown in Fig. 3. Figure 4(a) shows observed reflectivity changes at room temperature and at $5 \mathrm{~K}$. The round-trip time of the ultrasonic pulse echoes $\Delta t$ was measured to determine the out-of-plane elastic constant $C_{33}$;

$$
C_{33}=\rho v^{2}=\rho\left(\frac{2 d}{\Delta t}\right) .
$$

Here, $\rho$ and $d$ are mass density and film thickness, respectively. We considered that the mass density of current thin film is nearly equal to that of the bulk material. Two causes, volume defects and changes in the interatomic distance, could affect the mass density of the thin film. However, the XRD measurement shows that the fractional change in the interatomic distance is the order of $10^{-3}$, and its influence on the mass density is negligible. The other cause, volume defects, is also negligible because volume defects decrease the mass density and then would have increased the sound velocity, which has never been observed. Therefore, we used the bulk mass density of $2.145 \times 10^{4} \mathrm{~kg} / \mathrm{m}^{3}$ to determine the out-of-plane elastic constant $C_{33}$ of the $\mathrm{Pt}$ thin film. As a consequence, we obtained $C_{33}=379 \mathrm{GPa}$ at room temperature and $C_{33}=393 \mathrm{GPa}$ at $5 \mathrm{~K}$; The modulus increased by $3.53 \%$ with cooling down to $5 \mathrm{~K}$. Because of the strong (111) texture, we assumed the complete (111) textured Pt thin film, and compared the obtained stiffness with the longitudinal-wave modulus along [111] direction; $C^{<111>}=\frac{1}{3}\left(C_{11}+2 C_{12}+4 C_{44}\right)$ of bulk Pt is calculated to be $C^{<111>}=384.7 \mathrm{GPa}$ at room temperature and $C^{<111>}=391.6 \mathrm{GPa}$ extrapolated at $0 \mathrm{~K} .{ }^{17)}$ Thus, this modulus increase by $1.80 \%$ with cooling down to $0 \mathrm{~K}$. Therefore, the increment of the elastic constant of the Pt thin films at low temperature is significantly larger than that of bulk Pt by a factor of 2. The detailed mechanism is under investigation, but we attribute this observation to the high apparent temperature of thin films; The energy of thin films are inherently higher than the bulk materials due to the surface. Besides, the substrate restricts the lattice-parameter change, keeping the high vibrational amplitude of lattice, corresponding to apparently high temperature. Usual metallic materials show the saturation of the stiffness at low temperatures because of the quantum effect ${ }^{18)}$ (zero-vibration of the lattice), and the stiffness increment becomes smaller than the high-temperature region. The apparent high-temperature structure 
of thin film thus can cause larger increment of the stiffness even at low temperatures.

Next, we discuss the pulse width. Figure 4(b) shows Fourier spectra for the first echoes at each temperature. The spectrum becomes wider with cooling, which means that the shape of the pulse echo becomes narrow at low temperature. The pulse width of picosecond ultrasounds is affected by attenuation and electric resistance of the material. The increase of the former broadens the pulse, and that of the latter sharpens. For the usual metallic materials, electric resistance decreases with cooling since the primary factor of it is the thermal disturbance. Thus, this result indicates that Pt thin films show low attenuation for ultrasounds at low temperature. Theoretical calculation considering ultrasound attenuation and temperature dependence of electric resistance will let us extend the knowledge of pulse width.

\subsection{Si substrate with Al ultrathin film}

We deposited a $10 \mathrm{~nm} \mathrm{Al} \mathrm{film} \mathrm{as} \mathrm{the} \mathrm{transducer} \mathrm{material} \mathrm{for} \mathrm{ultrahigh-frequency} \mathrm{phonon} \mathrm{pulse}$ on the (001) surface of the Si substrate. Figure 5(a) shows observed reflectivity changes at room temperature and $5 \mathrm{~K}$. We clearly observe Brillouin oscillation from the Si substrate. Because the depth of penetration of the probe light influences the endurance of the Brillouin oscillation, the lower damping in the oscillation at the lower temperature indicates the decrement of optical absorption in Si. Figure 5(b) shows Fourier spectra for observed Brillouin oscillations. The relationship between the out-of-plane elastic constant $C_{33}$ and the frequency of the Brillouin oscillation $f$ is given by;

$$
C_{33}=\rho v^{2}=\rho\left(\frac{f \lambda}{2 n}\right) .
$$

The frequency at low temperature (broken line) was lower than at room temperature (solid line) by $2.20 \%$. However, it is noted that the elastic constants of Si increase at $5 \mathrm{~K}$ by 1.15 \%. ${ }^{19)}$ Thus, the decrease in the frequency of Brillouin oscillation indicates the decrease in the refractive index of $\mathrm{Si}$ at $5 \mathrm{~K}$ by $3.31 \%$. Using $n=5.57^{20)}$ at room temperature at $400 \mathrm{~nm}$, we obtain $n=5.39$ at $5 \mathrm{~K}$ at $400 \mathrm{~nm}$, which has never been reported. Thus, we can determine the temperature dependence of the refractive index of semiconductors using Brillouin oscillations.

\section{Conclusion}

Using PSLU method, we generated and detected picosecond acoustic phonon pulses in the thin film at various temperatures from room temperature to $5 \mathrm{~K}$. We observed multiple reflection echoes for Pt thin film and Brillouin oscillations from Si monocrystal substrate. These results indicate the larger increase of out-of-plane elastic constant $C_{33}$ for Pt thin films compared with bulk Pt and the decrease of the refractive index $n$ of Si at low temperature, respectively. It is also suggested that $\mathrm{Pt}$ thin films show low attenuation for ultrasounds at low temperature. 


\section{References}

1) H. Ogi, M. Fujii, N. Nakamura, T. Yasui, and M. Hirao : Phys. Rev. Lett. 98 (2007) 195503.

2) H. Ogi, M. Fujii, N. Nakamura, T. Shagawa, and M. Hirao : Appl. Phys. Lett. 90 (2007) 191906.

3) C. Thomsen, J. Strait, Z. Vardeny, H. J. Maris, J. Tauc, and J. J. Hauser : Phys. Rev. Lett. 53 (1984) 989.

4) C. Thomsen, H. T. Grahn, H. J. Maris, and J. Tauc : Phys. Rev. B 34 (1986) 4129.

5) O. B. Wright, B. Perrin, O. Matsuda, and V. E. Gusev : Phys. Rev. B 64 (2001) 081202.

6) A. Devos and C. Lerouge: Phys. Rev. Lett. 86 (2001) 2669.

7) O. Matsuda, O. B. Wright, D. H. Hurley, V. E. Gusev, and K. Shimizu : Phys. Rev. Lett. 93 (2004) 095501.

8) L. Parratt : Phys. Rev. 95 (1954) 359.

9) N. Nakamura, H. Ogi, and M. Hirao : Phys. Rev. B 77 (2008) 245416.

10) H. Ogi, T. Shagawa, N. Nakamura, and M. Hirao : Phys. Rev. B 78 (2008) 134204.

11) H. Ogi, T. Shagawa, N. Nakamura, M. Hirao, H. Odaka, and N. Kihara : Jpn. J. Appl. Phys. 48 (2009) 07GA01.

12) H. Tanei, N. Nakamura, Y. Kake, H. Ogi, K. Kusakabe, and M. Hirao : Jpn. J. Appl. Phys. 47 (2008) 3847.

13) H. Tanei, N. Nakamura, H. Ogi, and M. Hirao : Phys. Rev. Lett. 100 (2008) 016804.

14) H. Tanei, K. Tanigaki, K. Kusakabe, H. Ogi, N. Nakamura, and M. Hirao : Appl. Phys. Lett. 94 (2009) 041914.

15) B. J. Frey, D. B. Leviton, and T. J. Madison : Proc. SPIE, 6273 (2006) 62732J.

16) H.-Y. Hao and H. J. Maris : Phys. Rev. B 63 (2001) 224301.

17) R. E. Macfarlane, J. A. Rayne, and C. K. Jones : Phys. Lett. 18 (1965) 91.

18) Y. P. Varshni : Phys. Rev. B 2 (1970) 3953.

19) J. J. Hall : Phys. Rev. 161 (1967) 756.

20) D. F. Edwards : in Handbook of Optical Constants of Solids, ed. E. D. Palik (Academic Press, New York, 1985) p. 547. 
Figure 1 : Schematic drawing of developed optical system for low-temperature pump-probe measurement.

Figure 2 : X-ray total reflectivity measurement for Pt thin film on (001) Si substrate. Open circles denote measurements and solid line denotes the fitted theoretical function.

Figure 3 : X-ray diffraction measurement for Pt thin film on (001) Si substrate using CoK $\alpha$ radiation.

Figure 4 : Multiple reflection echoes of the longitudinal acoustic pulse for Pt thin film on (001) Si substrate observed at room temperature and $5 \mathrm{~K}$ (a) and Fourier spectra for the first echoes at each temperature (b).

Figure 5 : Brillouin oscillations from (001) Si substrate with Al ultrathin film observed at room temperature and $5 \mathrm{~K}$ (a) and their Fourier spectra (b). 


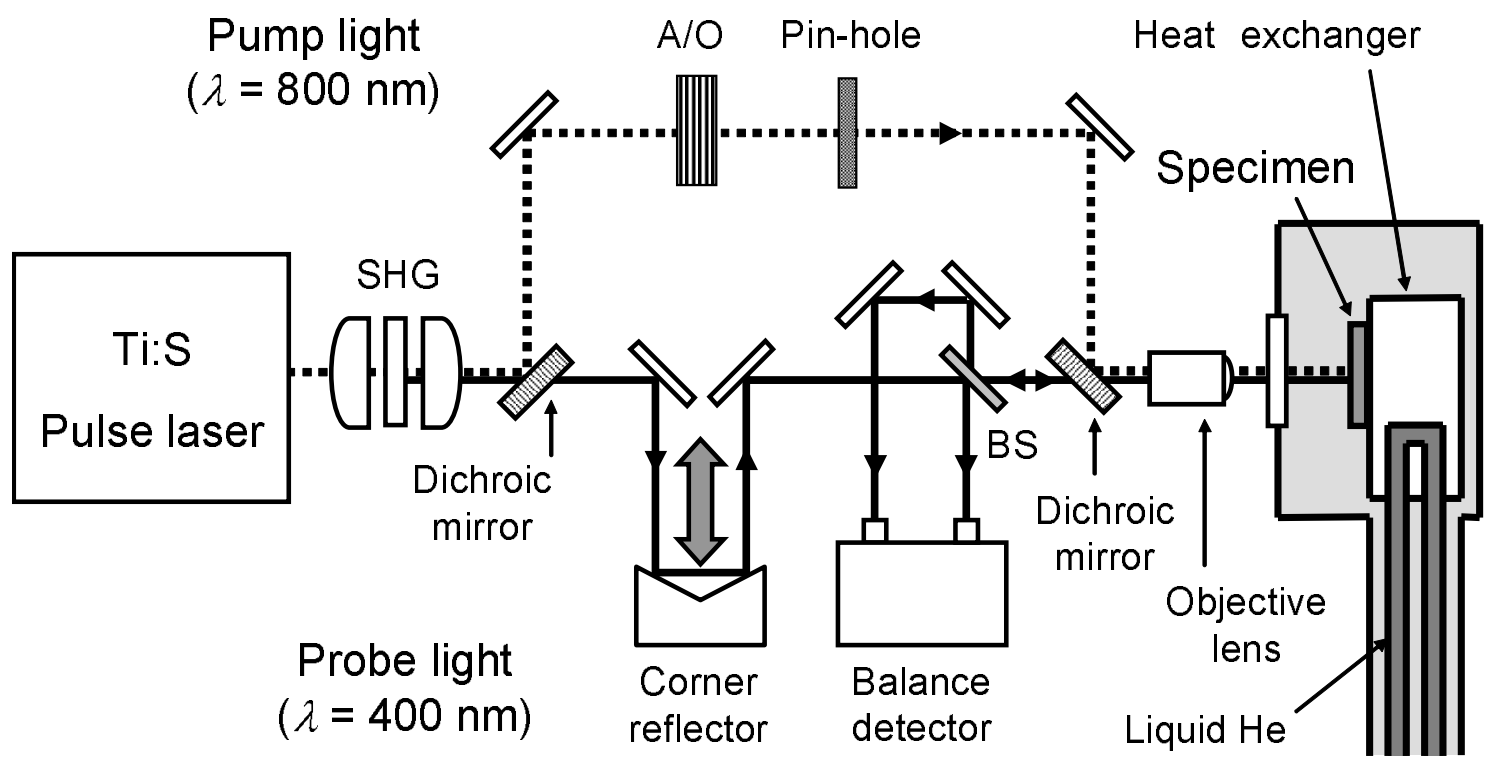

Fig. 1. 


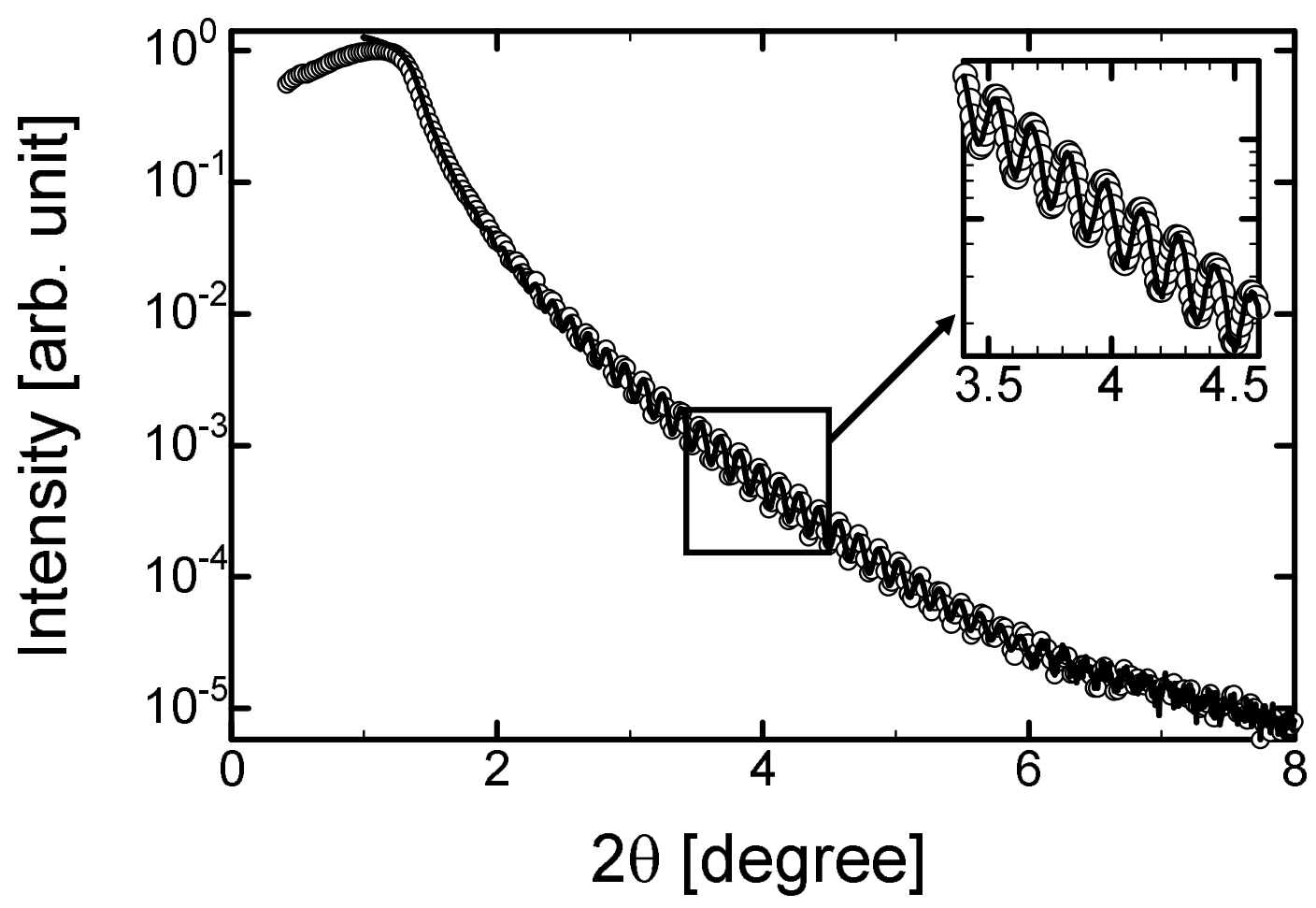

Fig. 2. 


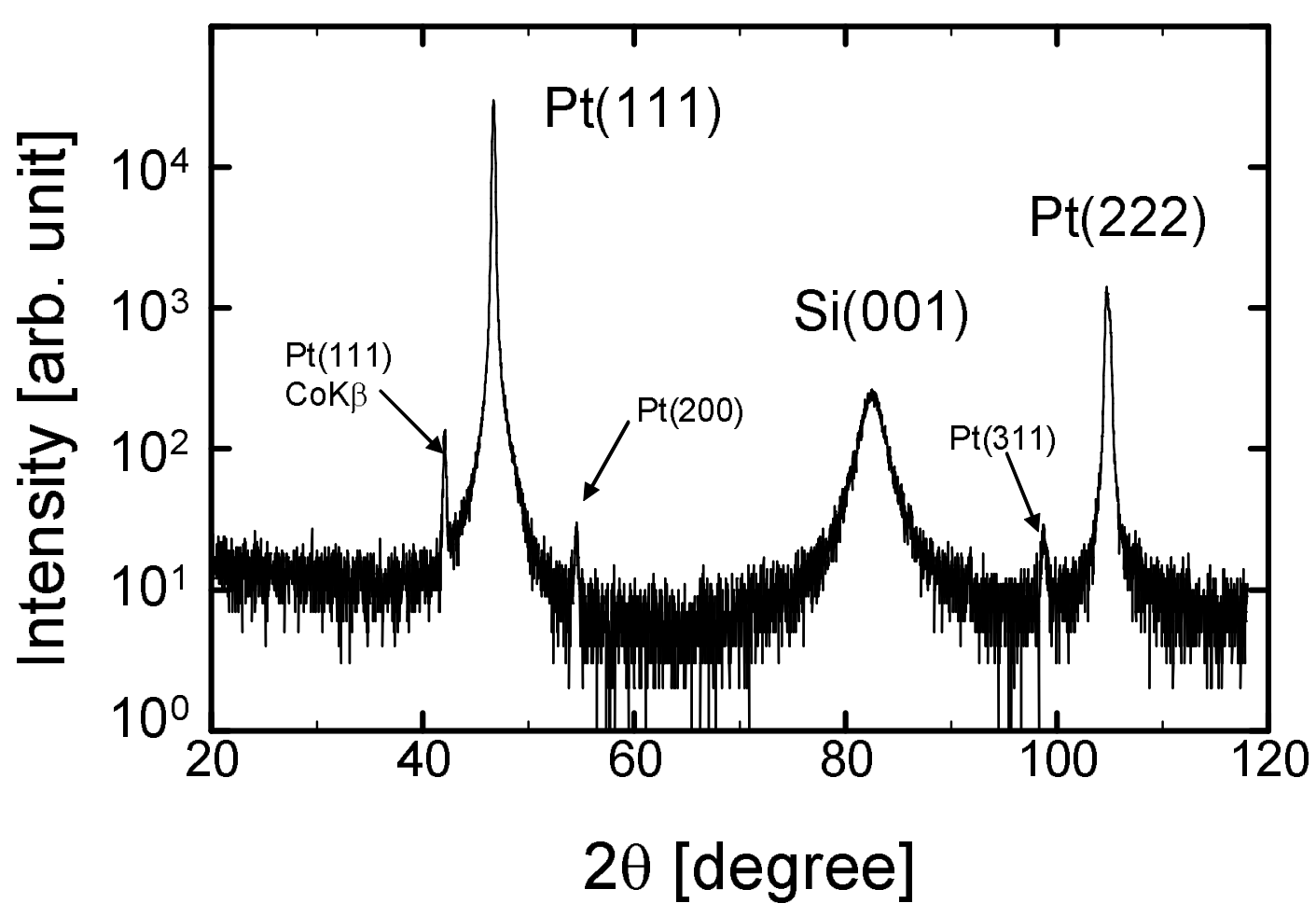

Fig. 3. 

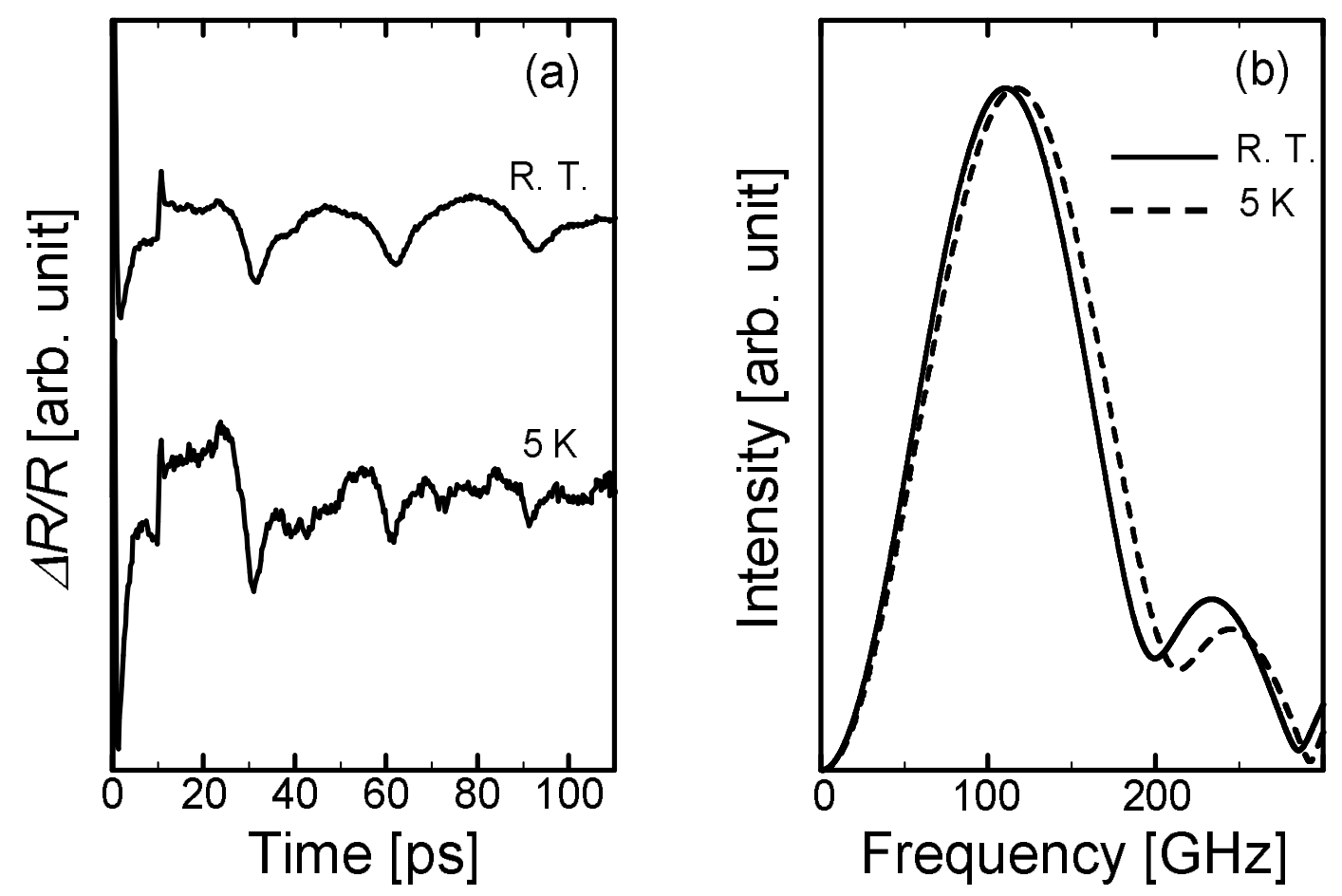

Fig. 4. 

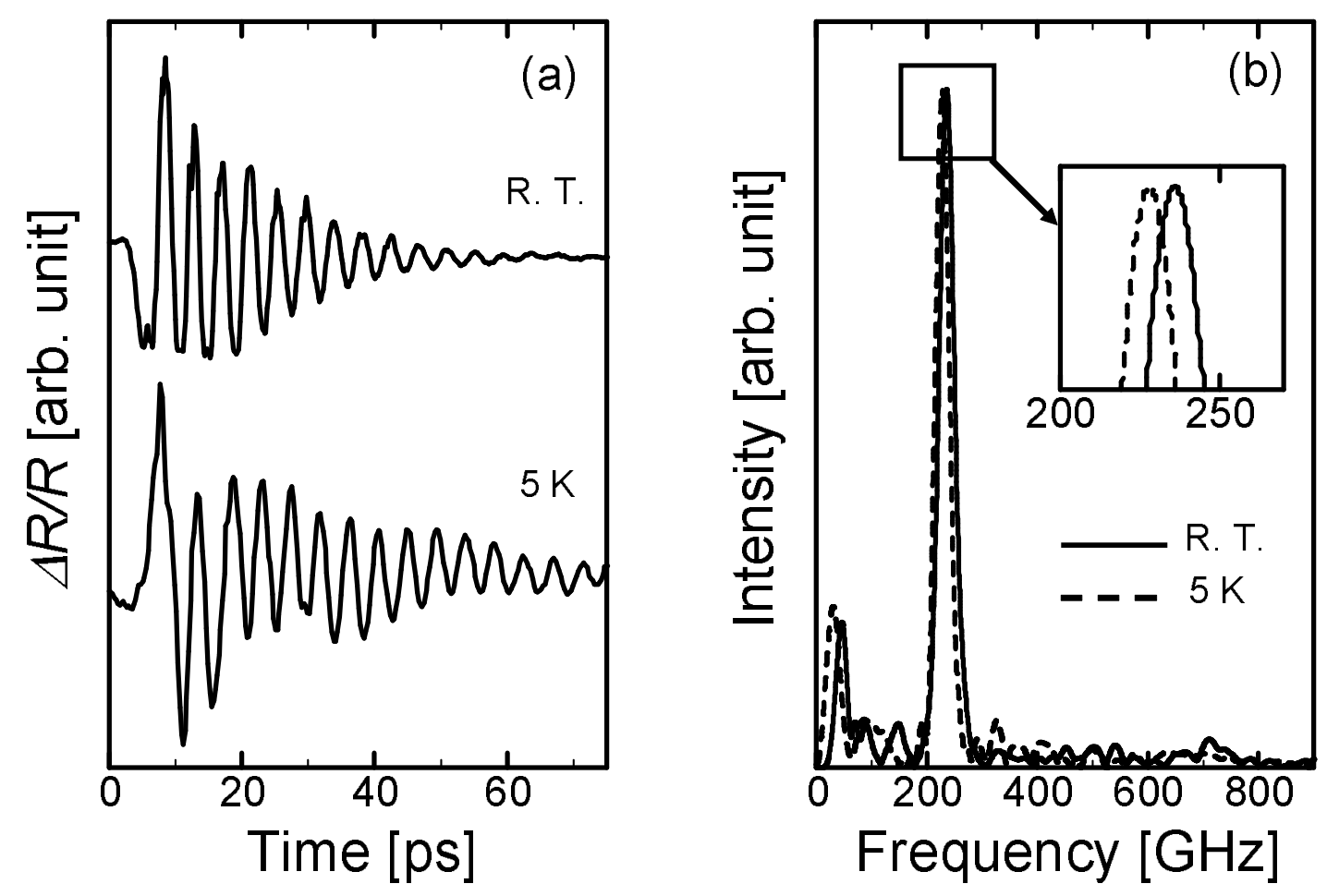

Fig. 5. 